
December 1934

\title{
AIR FORCES AND YAWING MOMENTS FOR THREE AUTOMOBILE MODELS
}

\author{
By Roy H. Heald
}

\section{ABSTRACT}

The lift, drag, cross-wind force, and yawing moment for three models representing automobiles having substantially different body shapes were measured in the wind tunnel. Measurements were made for relative wind angles, in the horizontal plane, between 0 and 180 degrees. The results of the model investigation, extrapolated to full scale, indicate the presence of rather large lift, lateral force, and yawing moment under certain conditions of car speed and natural wind speed.

\section{CONTENTS}

I. Introduction

II. Description of models and method of testing

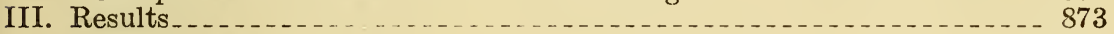

1. Force tests

2. Moment tests

IV. Discussion _...

V. Estimates of full-scale values.

vI. Conclusion

\section{INTRODUCTION}

The early measurements of the air forces on automobile models in wind tunnels were confined, largely, to the measurement of the air resistance at the zero degree angle of yaw. Generally speaking the purposes of such tests were to find shapes of low air resistance and to furnish a basis for estimating the magnitude of the full-scale resistance due to motion in still air. Recently attention has been directed also to the effects of side winds., ${ }^{1,2}$

The system of aerodynamic forces which acts on an automobile moving relative to the air is analogous to that which acts on an aircraft in flight. These forces arise as the resuit of local differences in air pressure, the net effect of the local pressures being to give rise to a tendency to motion in a certain direction. The magnitude and direction of this tendency can be represented by a single resultant force. In rectilinear motion through still air, or in horizontal head or tail winds, the resultant of the air forces acts in the vertical plane of symmetry of the vehicle. In this case the resultant may be defined in terms of lift, drag, and pitching moment about some fixed point in the vehicie such as the center of gravity. The introduction

1 BS J. Research 11, 285 (1933) RP591.

2 Auto. Indus. 71, 172 (1034). 
of a quartering horizontal wind gives rise to a relative wind which acts at an angle to the direction of motion, somewhat less than the angle of the natural wind.

As a consequence of the new set of conditions, there is no plane of symmetry and 3 forces and 3 moments are necessary to define the resultant completely. Choosing axes with origin at the center of gravity, the three forces are the longitudinal force, in the direction of motion of the car (positive when opposing the motion), the lateral force, in a horizontal direction at right angles to the longitudinal force (positive when to the left looking forward), and the lift (positive when directed upward). The three moments are the pitching mo-
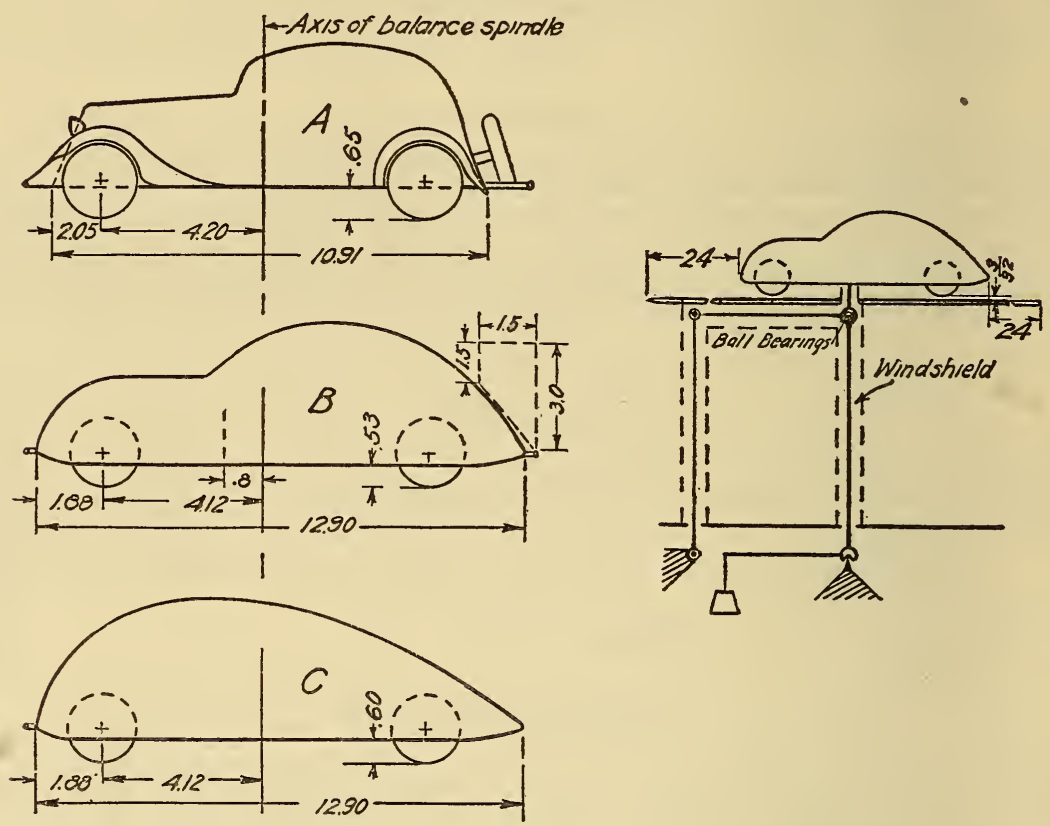

FIGURE 1.-Models used in making force and yawing moment measurements.

The scale was about 1:15. The dimensions are given in inches. The sketch at the rightindicates the method used in measuring the drag and cross-wind forces.

ment about the lateral axis, the rolling moment about the longitudinal axis, and the yawing moment about the vertical axis.

Because of the interest in the limited data on the longitudinal and lateral forces reported in the Bureau's Research Paper 591, and because it appeared desirable to obtain further information on the models exhibited at the Century of Progress Exposition, a more extensive series of measurements has been made on these models, including the measurement of lift and yawing moment as well as longitudinal and lateral forces. The present paper describes the experiments.

\section{DESCRIPTION OF MODELS AND METHOD OF TESTING}

Outline drawings showing the principal dimensions of the models used for the experiments are given in figure 1 . The models were those used in the tests previously reported, ${ }^{3}$ models $\mathrm{A}, \mathrm{B}$, and $\mathrm{C}$ 
corresponding respectively to models 4,5 , and 6 of the earlier tests. In the earlier tests, measurements were made only for the "head-on" wind condition, a model being suspended by four wires in the wind tunnel with its wheels just clearing the surface of the ground plane. The displacements due to the wind were measured for a number of speeds and the drag computed from the displacement, the swinging radius, and the weight. A correction was applied for the resistance of the wires.

The method of measuring the forces on the models in the present investigation is indicated in figure 1 . The measurements were made using an NPL type balance, care being taken to shield all exposed parts of the balance and fittings in order to reduce the spindle correction to a minimum. A parallelogram linkage attached to the balance in the 54-inch wind tunnel was used for making the drag and cross-wind force measurements. Such an arrangement obviated the necessity of determining the center of pressure of the model and spindle combination but did not permit simultaneous observations of drag and cross-wind force. The lift and yawing-moment measurements were made using the regular equipment of the NPL balance, the lift being determined by means of the vertical lift attachment of the balance and the yawing moment by means of the standard torque balance. ${ }^{4}$

Previous tests ${ }^{5}$ having shown a marked effect of the shape of the leading edge of the ground plane on the resistance of a streamline model, an investigation was made of the velocity distribution over a platform extending entirely across the air stream, which was used to represent the ground. The faired section of the upstream edge of the platform is indicated in figure 1. The mean air speed at the test section, 2 feet downstream from the leading edge of the ground plane, was determined from a traver'se of an area 6 inches square in the plane of the balance spindle, observations being made at some 30 stations. The air speed was found to be substantially uniform down to within one-half inch of the ground plane. It would be expected therefore that the effects on the model arising as a result of the interaction of the boundary layer of the platform with the air flow around the model would be confined to the region of the wheels since the lower surface of each model, as mounted in the test position, was some half inch above the ground plane when the wheels were just clear.

\section{RESULTS}

\section{FORCE TESTS}

The experimental arrangement simulated the conditions encountered when an automobile is driven in a quartering horizontal wind, the direction of the air stream in the wind tunnel representing the direction of the relative wind resulting from the natural horizontal wind and the motion of the car. While the natural wind may have a vertical component, the magnitude of that component is probably very small within the height of an automobile above the ground. It was convenient to measure the components of the resultant force along and perpendicular to the direction of the relative wind, i.e., the drag and cross-wind force, and to compute from them the compo-

Tech. Rep. 72 Nat. Adv. Comm. Aeron.
J J. Research NBS 13, 863 (1934) RP748. 
nents along and perpendicular to the normal direction of motion of the car, i.e., the longitudinal and lateral forces.

Measurements were made at intervals of $10^{\circ}$ from 0 to $180^{\circ}, 0^{\circ}$ corresponding to a head-on wind, $180^{\circ}$ to a tail wind. The net observed force, $F$, in pounds, was divided by the product of $A$, the maximum frontal area, projected normal to the longitudinal axis
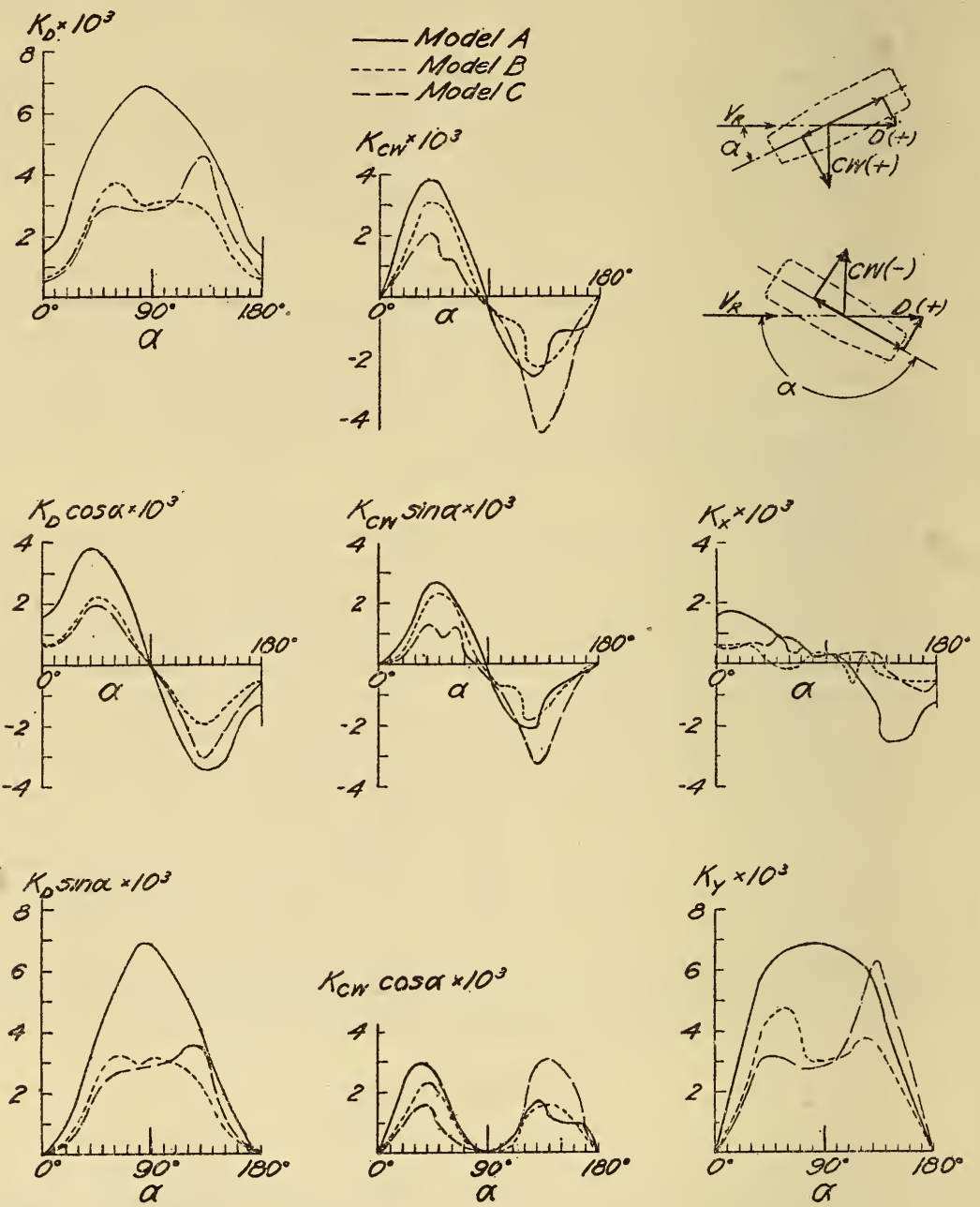

FIGURE 2.-Resolution of the drag and cross-wind force coefficients to give longiiudinal-and lateral-force coefficients.

of the model, in square feet, and the square of the relative air speed, $V_{\mathrm{R}}$, in miles per hour, to obtain a coefficient, $K$.

Thus $K=\frac{F}{A V_{R}^{2}}$. Subscripts are used to denote the several components, $D$ for drag, $C W$ for cross-wind force, $X$ for longitudinal force, $Y$ for lateral force, $L$ for lift. The relations between drag and cross-wind force coefficients, and longitudinal and lateral force coefficients are as follows: 


$$
\begin{aligned}
& K_{x}=K_{D} \cos \alpha-K_{C W} \sin \alpha \\
& K_{y}=K_{D} \sin \alpha+K_{C W} \cos \alpha
\end{aligned}
$$

where $\alpha$ is the angle of yaw, corresponding to the angle of relative wind in the full-scale case.

The longitudinal and lateral force coefficients are plotted in figure 2 , and as a matter of interest, the contributions of the drag and crosswind force to the longitudinal and lateral force are also plotted.

The positive directions of the drag and cross-wind force are indicated in figure 2 . The longitudinal force is considered positive when opposite to the direction of motion of the car; when positive, the longitudinal force retards the car; when negative, it aids the motion of the car. The lateral force is considered

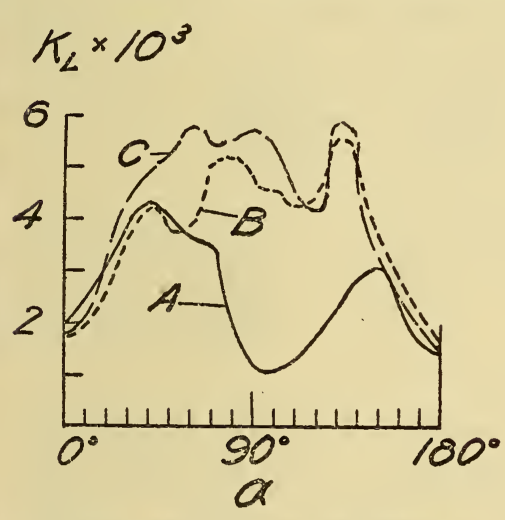

Figure 3.-Lift coefficients.

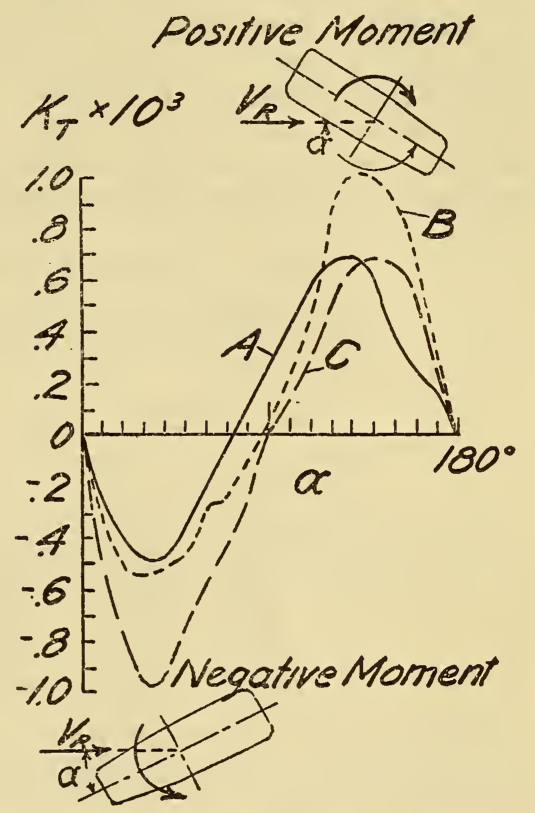

FIGURE 4.-Yawing moment coefficients.

positive when directed to the left, looking forward. The lift coeffcients, plotted in figure 3 , are considered positive when the lift is directed upward.

\section{MOMENT TESTS}

The axis about which most of the yawing moments were measured was half-way between the front and rear axles. (Axis of balance spindle in figure 1.) The yawing moment, $M$, in pounds-feet, was divided by the product of $A V_{R}^{2}$, and $l$, the overall length of the model, in feet, to give the yawing moment coefficient $K_{T}$, plotted in figure 4 . The direction of yawing moment is considered positive when the moment tends to reduce the angle between the longitudinal axis of the body and the direction of the relative wind.

Generally speaking, streamline bodies moving in still air, without interference from surrounding objects, are directionally unstable in an aerodynamic sense, i. e., when yawed with respect to the direction of motion the tendency of the resulting moment is to increase the yaw. The degree of instability is partly dependent on the magnitude and shape of the exposed areas ahead of and behind the center of 
gravity and it might be expected that the streamline models in the presence of the ground plane would exhibit somewhat the same characteristics. The moment curves in figure 4 show that all three models are aerodynamically unstable when the vertical plane containing the center of gravity is assumed to be half-way between the front and rear axles, an assumption believed to be fairly representative of actual cases. Obviously there are two methods of improving stability: (1) by moving the center of gravity forward, and (2) by adding effective area behind the center of gravity as, for example, by the use of fins. A few experiments dealing with the question of stability were made and for these, model $B$, figure 1 , was selected. It will be noted from table 1 that the model was brought into approximately neutral condition, for the case of relative wind from the forward quarter, by using three fins of equal areas, uniformly spaced laterally. The total area of the fins represented about 90 percent of the maximum projected frontal area of the model.

TABLE 1.-Stability measurements (model B, fig.1)

Values of $K_{T} \times 10^{3}$ for various angles of relative wind $(\alpha)$.

\begin{tabular}{|c|c|c|c|c|}
\hline$\alpha$ & $\begin{array}{l}\text { Without } \\
\text { fins }\end{array}$ & $\begin{array}{c}\text { With } 3 \\
\text { fins }\end{array}$ & $\begin{array}{l}\text { Without fins; } \\
\text { center of mo- } \\
\text { ments moved } \\
\text { ahead } 0.8 \\
\text { inch }\end{array}$ & $\begin{array}{l}\text { With } 3 \text { fins; } \\
\text { center of mo- } \\
\text { ments moved } \\
\text { ahead } 0.8 \\
\text { inch }\end{array}$ \\
\hline $\begin{array}{r}\text { Degrees } \\
0 \\
10 \\
20 \\
30 \\
50 \\
70\end{array}$ & $\begin{array}{l}0.00 \\
-.32 \\
-.51 \\
-.54 \\
-.43 \\
-.27\end{array}$ & $\begin{array}{r}0.00 \\
+.07 \\
-.04 \\
+.04 \\
+.05 \\
+.25\end{array}$ & $\begin{array}{r}0.00 \\
-.28 \\
-.43 \\
-.41 \\
-.17 \\
-.13\end{array}$ & $\begin{array}{r}0.00 \\
+.09 \\
+.15 \\
+.33 \\
+.33 \\
+.58\end{array}$ \\
\hline
\end{tabular}

Moving the assumed position of the center of gravity (axis of moments) ahead a distance equivalent to about 1 foot; full scale, substantially reduced the unstable moment within the range of angles investigated. The model was made completely stable, as regards wind from the forward quarter, by a combination arrangement which consisted in moving the assumed position of the center of gravity ahead 0.8 inch (about 1 foot, full scale) and using the three fins. It may be noted in this connection, however, that whatever is gained by the use of fins, as regards stability with respect to wind from the forward quarter probably represents a corresponding loss with respect to winds from the rear quarter.

\section{DISCUSSION}

A noteworthy result of the tests is to indicate the behavior of the longitudinal force coefficient in the case of the streamlined models and, in particular, model B. The coefficient changes sign twice in the first quadrant and three times in the second quadrant. Although the longitudinal force coefficient was obtained, by computation, as the difference of two larger quantities, as indicated in figure 2, the presence of experimental errors amounting to several percent in the original observations would be required to account for this behavior. It appears more probable that the changes were caused by the alternate preponderance of the drag and cross-wind components as the angle of yaw was increased. 
Another outstanding characteristic common to all three models is the occurrence of comparatively large lateral forces. In the first quadrant both streamline models were found to have lateral forces of the order of one-half the lateral force on the model of the conventional car. In the latter part of the second quadrant, however, the lateral force on streamline model $\mathrm{C}$ exceeded the lateral force on the model of the conventional car.

Between 0 and $40^{\circ}$ yaw the lift of all three models was of about the same order of magnitude. In the neighborhood of the broadside position, however, the lifts of both faired models were found to be considerably greater than the lift of the model of the conventional car. A relationship of this kind between the lift forces might be expected as a result of the somewhat greater curvature in lateral section of the streamline models.

The yawing moment curves of the three models exhibit the same general characteristics. All were found to be unstable for the position originally assumed for the center of gravity. The torque measurements made with several difierent fin combinations on model B indicate that a total fin area of approximately 90 percent of the maximum projected frontal area would be required to bring the full scale car to a stable condition with respect to relative winds from the forward quarter.

\section{ESTIMATES OF FULL-SCALE VALUES}

The equations for determining the magnitude, $V_{R}$, and the direction, angle $\alpha$, of the relative wind, having given the magnitude, $V_{w}$ and the direction, angle $\beta$, of the natural wind and the velocity of the vehicle, $V_{A}$, are:

$$
\begin{gathered}
V_{R}=\sqrt{V_{A}{ }^{2}+V_{w}{ }^{2}+2 V_{A} V_{w} \cos \beta} \\
\alpha=\sin ^{-1} \frac{V_{w} \sin \beta}{V_{R}}
\end{gathered}
$$

The magnitudes of the forces and torques are dependent on the velocity and angle of the relative wind. These are dependent, in turn, on the velocity of the vehicle, $\left(V_{A}\right)$, the velocity, $\left(V_{w}\right)$, and the direction, angle $\beta$, of the natural wind which is here considered to act in the horizontal plane. Thus there are an indefinite number of combinations of car velocity, natural wind velocity, and natural wind direction that can occur, each combination corresponding to a relative wind whose magnitude and direction can be computed. ${ }^{6}$ Certain combinations give rise to a relative wind at $90^{\circ}$ to the direction of motion and in some cases the angle of the relative wind may be greater than $90^{\circ}$ to the direction of motion, as for example, when the natural wind blows nearly from the rear at a speed greater than that of the car.

In order to illustrate the orders of magnitude of the full-scale forces the values given in table 2 have been computed for full-scale replicas of models $A, B$, and $C$, figure 1, assuming a driving speed of 50 miles per hour. The coefficients shown in figures 2,3 , and 4 were used in the computation assuming a frontal area, previously defined, of 25 square feet and a body length of 15 feet. The forces given in

6 J. Soc. Auto. Engrs., 33, 18, (November 1933). 
the table have been rounded to the nearest 10 pounds, the moments to the nearest 10 pounds-feet. The effects of scale and of projections, such as the undercarriage, present on the full-scale car but not represented on the model, cannot be predicted accurately. The assumed driving speed is believed to be representative. Driving in continuous winds of 10 miles per hour is common and gusts of 30 to 40 miles per hour may be encountered.

TABLE 2.-Forces and yawing moment for full-scale replicas of the models, scale effect neglected

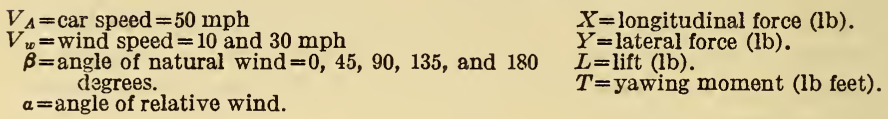

$V_{w}=10 \mathrm{mph}$

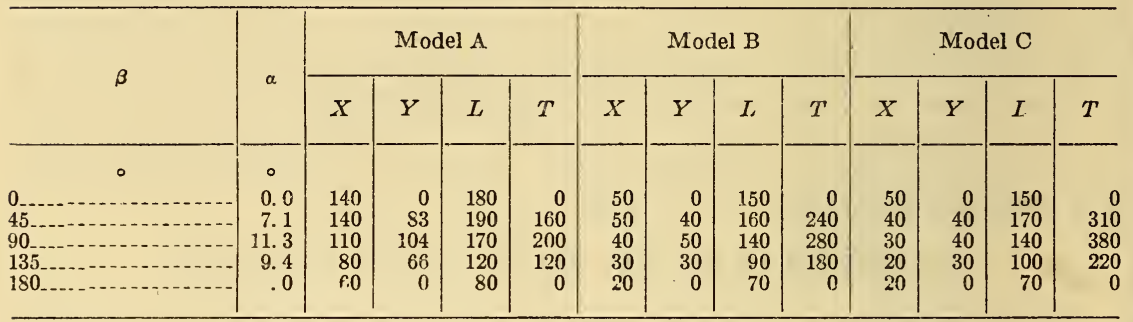

$V_{w s}=30 \mathrm{mph}$

\begin{tabular}{r|r|r|r|r|r|r|r|r|r|r|r|r|r}
\hline 45 & 0.0 & 240 & 0 & 320 & 0 & 100 & 0 & 270 & 0 & 100 & 0 & 270 & 0 \\
90 & 16.6 & 230 & 350 & 400 & 550 & 90 & 170 & 340 & 770 & 80 & 110 & 370 & 1120 \\
135 & 31.0 & 130 & 410 & 340 & 470 & 50 & 250 & 310 & 570 & 60 & 200 & 370 & 1000 \\
180 & 50 & 190 & 140 & 180 & 10 & 120 & 130 & 210 & 20 & 90 & 150 & 370 \\
0 & 0 & 20 & 0 & 20 & 0 & 10 & 0 & 20 & 0 & 6 & 0 & 20 & 0 \\
\hline
\end{tabular}

\section{CONCLUSION}

Broadly speaking the aerodynamic problem placed before the designer is to find the vehicle body shape requiring the minimum amount of power to overcome the air forces and having satisfactory stability. From this viewpoint it is necessary to consider the action of all forces which arise as a result of relative motion between the full-scale automobile and the air.

Bearing in mind the limitations imposed on the model method, the results of the present experiments indicate that substantial reduction in the longitudinal and lateral forces, throughout the 0 to $180^{\circ}$ range of relative wind angles, is to be expected from the use of streamline body forms. However, the lift forces and yawing moments may be somewhat greater for certain ranges of angles of relative wind in the case of streamline body forms. The results of the extrapolation from the model measurements to full-scale point to the possibility of rather large lateral forces and yawing moments for all three types of bodies at high car and wind speeds.

Washington, August 24, 1934. 